Portland State University

PDXScholar

6-8-2018

\title{
Early Life Sleep Disruption Alters Dendritic Spine Density and Morphology in Prairie Voles
}

Alex Q. Chau

Portland State University

Follow this and additional works at: https://pdxscholar.library.pdx.edu/honorstheses

Let us know how access to this document benefits you.

\section{Recommended Citation}

Chau, Alex Q., "Early Life Sleep Disruption Alters Dendritic Spine Density and Morphology in Prairie Voles" (2018). University Honors Theses. Paper 557.

https://doi.org/10.15760/honors.563

This Thesis is brought to you for free and open access. It has been accepted for inclusion in University Honors Theses by an authorized administrator of PDXScholar. Please contact us if we can make this document more accessible: pdxscholar@pdx.edu. 
Early life sleep disruption alters dendritic spine density and morphology in prairie voles

by

Alex Q. Chau

An undergraduate honors thesis submitted in partial fulfillment of the requirements for the degree of

Bachelor of Science

in

University Honors

and

Biology

Thesis Advisor: Miranda M. Lim, MD, Ph.D.

Co-thesis Advisor: Carolyn E. Jones, Ph.D.

Portland State University

2018 


\section{ABSTRACT}

Synaptic pruning within neurons in the brain during development allows for maintenance of proper neuronal connections and the elimination of aberrant ones. Rapid eye movement (REM) sleep is critical for pruning and maintaining new synapses formed during both development and learning. We hypothesize that disrupting REM sleep early in life will result in long lasting changes in synaptic density in cortical brain regions. The prefrontal cortex (PFC) is a late-maturing region that modulates higher order social and cognitive functions. Abnormally high dendritic spine density in the PFC is implicated in neurodevelopmental disorders such as autism spectrum disorder (ASD). Emerging research in our lab suggests that selectively suppressing REM sleep early in life in the socially monogamous prairie vole (Microtus ochrogaster) impairs social development and increases inhibitory interneurons in the PFC, consistent with ASD pathology. Using Golgi-Cox staining in adult prairie vole post-mortem tissue, we quantified dendritic spines in the prefrontal cortex in adult animals that underwent early life sleep disruption (ELSD). In males, ELSD increased spine density and decreased spine width selectively in the apical oblique distal (>90 $\mu \mathrm{m})$ segments of pyramidal neurons in prelimbic cortex layers II/III. Distal dendrites reflect long range inputs from further cortical and thalamic regions, suggesting that ELSD may lead to an impaired ability to integrate sensory information. Ongoing work will examine dendritic spine density and morphology earlier in development and in additional brain regions, including the primary somatosensory cortex and other layers of the PFC. Results from these studies will enhance our understanding of how modulation of sleep early in life contributes to the neuropathology of developmental disorders. 


\section{INTRODUCTION}

Humans and other mammalian species have very high levels of REM sleep early in development, followed by a slow decrease to adult levels (Roffwarg, Muzio, and Dement 1966; Siegel, 2005). The "ontogenetic hypothesis" of sleep postulates that early developmental REM sleep is important for brain patterning (Roffwarg, Muzio, and Dement 1966). Roffwarg and colleagues proposed that sleep with rapid eye movements (REM) supplies endogenous stimulation to the developing brain, providing excitation to higher functional centers necessary for cortical development (Roffwarg, Muzio, and Dement 1966). Indeed, a number of neurodevelopmental disorders are associated with poor sleep early in life.

Over a century ago, Ramon y Cajal proposed that all brains were made up of neurons that communicated with each other through junctions called synapses. We now know that synaptic transmission is a biological process by which a neuron communicates with a target cell across a synapse. In response to an action potential or graded electrical potential, neurotransmitters (e.g. glutamate) are released from the presynaptic neuron and bind to and activate specific receptors on the post-synaptic neuron. The maturation of cortical circuits in many species is characterized by an initial period of massive synaptogenesis, followed by the selective elimination of excitatory synapses during adolescence (Rakic, Bourgeois, and Goldman-Rakic 1994). During the pruning process, the axons of neurons are degenerated or retracted, resulting in synapse elimination. Synaptic pruning is thought to be necessary for proper maturation and function of the mature synapses (Nimchinsky et al., 2002). These changes in synaptic connections are regulated by sensory experience, widely thought to reflect learning 
processes (Bailey and Kandel, 1993; Buonomano and Merzenich, 1998). It has also been suggested that synaptogenesis and pruning during development could be modulated by sleep and wakefulness (Tononi and Cirelli 2006). Tononi and Cirelli hypothesized that pruning of dendritic spines occurs during sleep, ensuring the maintenance of balanced synaptic input to cortical neurons. This remodeling of neural circuits corresponds to a sensitive period for the pathophysiology of neurodevelopmental disorders, including autism spectrum disorder (ASD) (Paus, Keshavan, and Giedd 2008).

Dendritic spines are micron-sized protrusions of the dendritic shaft that serve as the postsynaptic component of most excitatory synapses in the central nervous system (Gray 1959). They are found on excitatory neurons including glutamatergic pyramidal neurons of the neocortex and hippocampus. Spine modifications determine the strength and stability of the synaptic transmission in learning, memory, and behavioral functions (Haws et al. 2014; Sala and Segal 2014). The dendritic spine densities on these neurons represent the number of excitatory inputs, which varies between species and regions. The density of dendritic spines has direct implications for the functionality and connectivity of the cell. Synapses tend to be located at the heads of dendritic spines, therefore higher spine densities would suggest a higher density of synapses. The shapes and sizes of dendritic spines are highly plastic, typically $0.5-2$ microns in length (Harris and Kater 1994). Spine morphology is classified as thin, stubby, mushroom, filopodia, and branched (Chang and Greenough 1984; Risher et al. 2014). Spines are classified into specific morphologies based on the spine's head to neck diameter ratio. Spine morphology is associated with function. Mushrooms spines are stable, long-lived 
and form strong excitatory synapses (Trachtenberg et al. 2002; Kasai et al. 2003) while thin spines are immature, unstable, short-lived, and form weak excitatory synapses (Rochefort and Konnerth 2012).

Past studies have shown that the density and size of dendritic spines are regulated by a variety of genetic, epigenetic, and environmental factors, highlighting the dynamic nature of spines and their importance to brain plasticity and function (Harris and Stevens 1989). The work of Hutsler and Zhang on dendritic spines on Golgi-Cox stained pyramidal cells in the frontal, temporal, and parietal regions of ASD subjects showed greater spine densities on the pyramidal apical dendrites within the most superficial layer (layer II) of each cortical region. Higher spine densities were associated with decreased brain weights, but showed no relationship to adult brains (Hutsler and Zhang, 2010). Work in adolescent mice support Tononi and Cirelli's hypothesis that changes in dendritic spine density are influenced by the wake or sleep state, showing that waking results in an increase of cortical spines, whereas sleep is associated with spine loss (Maret et al. 2011). However, it remains poorly understood how spine pathology occurs, and how it is associated with the onset and progression of ASDrelated symptoms. Additional animal models and human studies on dendritic spine density and spine morphology in postmortem tissue are needed to understand the properties and mechanisms of these structures in the cerebral cortex of patients with autism.

Prairie voles (Microtus ochrogaster) are a highly social rodent species that form lifelong pair bonds with other individuals. We used this model organism to explore the neural circuitry governing sleep ontogeny and examined how sleep deprivation during 
early development affects species-typical cognitive and social behaviors during the juvenile stage and adulthood. To examine whether sleep during a critical period promotes proper brain development, we focused on pair-bonding because it is a robust, innate behavior with well-mapped circuitry. We found that early life sleep disruption prevented pair bond formation in male prairie voles, suggestive of an ASD behavioral phenotype. The prefrontal cortex (PFC) is involved in the neural circuitry of pair bond formation in prairie voles (McGraw and Young 2011) and is necessary for behaviors associated with higher cognition (Yizhar et al. 2011). We propose that disrupting REM sleep early in life will result in changes in synaptic density of the PFC contributing towards an ASD behavioral phenotype.

\section{MATERIALS AND METHODS}

\section{Animals and Early Life Sleep Disruption}

Experiments were conducted on laboratory-reared prairie voles (Microtus ochrogaster). Studied prairie voles were born in our colony and maintained in their standard home cages under 14:10 hour light/dark cycle with food and water available ad libitum. All experimental procedures were carried out in accordance with the National Institutes of Health Guide for the Care and Use of Laboratory Animals and with the approval of the Portland VA Research Foundation Institutional Animal Care and Use Committee. Voles received either one week of early life sleep disruption or control conditions during post-natal week 3 . We utilized a method of sleep disruption using an orbital rotor shaker with a repeated cycle of $10 \mathrm{sec}$ on, $100 \mathrm{sec}$ off continuously across 
one week controlled by a timer and validated by EEG recordings. Housing on the orbital shaker dramatically and selectively decreased the amount of REM sleep, but not NREM sleep, and only moderately decreased total sleep time. This method of sleep disruption is advantageous because vole pups can remain housed with their natal litter and both parents, essentially undisturbed other than the sleep disruption. In our lab, voles maintained in these conditions showed no changes in serum corticosterone or body weight across development, consistent with studies validating this method of sleep disruption in mice (Li et al., 2014). Following one week of early life sleep disruption, we conducted histological studies to examine the dendritic spine density and morphology in adult (P77) prairie voles.

\section{Golgi-Cox Staining}

We performed the Golgi-Cox method on adult brain tissue after either ELSD or control conditions from P14-P21 to quantify the dendritic spine density and spine morphology in regions implicated in ASD, including the PFC. Voles from the two treatment groups were euthanized with an overdose of isoflurane, were rapidly decapitated and had their brains removed. Brains were bisected sagittally and only one half the brain was used for analysis. Brain tissue was stained using the FD Rapid Golgi Stain Kit (PK401, FD Neurotechnologies, Columbia, MD). Tissue slices were impregnated in chromate mixture of "Solution A" (potassium dichromate and mercuric chloride) and "Solution B" (potassium chromate) for 9 days (changing the solution once after $24 \mathrm{~h}$ ) in low ambient light, before being transferred into cutting solution "Solution C". Brains were sectioned on a vibratome at $200 \mu \mathrm{m}$ and then mounted on gelatin- 
coated slides. Once dry, slides were rinsed in diH2O and developed in "solution D", dehydrated in ethanol gradients, and cleared in xylenes before cover slipping with Permount ${ }^{\mathrm{TM}}$ Mounting Medium (Fisher Scientific Co., Waltham, MA). Once dry, slides were then cleared in xylene solution and stored in darkness until further imaging and analysis. All slides were assigned a numerical code to conceal treatment group; thus experimenters were blinded to condition during both imaging and dendritic spine quantification.

\section{Image Acquisition}

Pyramidal cells from layer II/III of the medial PFC (prelimbic cortex and infralimbic cortex) were selected for study and located using the Paxinos and Franklin stereotaxic atlas for laboratory mice as a reference (Paxinos and Franklin 1997). Pyramidal neurons were identified by their characteristic triangular soma-shape, apical dendrites extending toward the pial surface and numerous dendritic spines. This step was performed under bright-field illumination on a Leica DFC365 FX microscope and Neurolucida 11.07 (MBF Bioscience, Williston, VT USA) program by an observer blinded to experimental condition and sex. The following criteria were used to select pyramidal dendrites for imaging: (1) fully impregnated and (2) not obscured by overlapping dendrites or large staining debris. If the dendrite met the criteria, apical secondary and tertiary dendrites of these neurons were selected for analysis. Each tissue slice was initially viewed under low $5 x$ magnification to establish the region of interest (layers II/III of the prelimbic and infralimbic cortex). Next, a pyramidal cell dendrite within the region of interest was viewed at $40 x$ magnification to determine 
whether the dendrite fulfilled the above criteria. Z-stacks image of Golgi-stained dendrites ( $80 \mu \mathrm{m}$ total on the Z-axis; optical section thickness $=2 \mu \mathrm{m} ; 41$ images per stack; image size, $2,048 \times 1535$ pixels $(0.1774 \times 0.1774 \times 2 \mu \mathrm{m})$ were taken at $40 \mathrm{x}$ magnification using the Neurolucida program. 2-3 pyramidal cells from the prelimbic region were imaged for each tissue slice whenever staining permitted. From each tissue slice, 2 or more cells were imaged and analyzed. We obtained neocortical tissue from males ( $n=4-6$ animals/group) and females ( $n=3-5$ animals/group).

\section{Dendritic Spine Quantification}

Each image stack was extracted using ImageJ software (NIH, Bethesda, MD) and subsequently imported into RECONSTRUCT software (Fiala, 2005) for analysis as described in (Risher et al. 2014). All images are processed using ImageJ Software. Apical oblique secondary and tertiary dendritic branches and apical tufts of pyramidal neurons in each region were analyzed, measuring the length and width of each protrusion with visible connections to the dendritic shaft from dendritic segments 10-20 $\mu \mathrm{m}$ in length. Because spine density and possibly spine size changes as a function of distance from the soma (Benavides-Piccione et al., 2002), we compared similar segments of dendrites between different pyramidal neurons, selecting segments of apical dendrites which were located at the same proportional distance from the soma. We selected apical dendrites segments that were $40-200 \mu \mathrm{m}$ distant from the soma. Every attempt was made to sample equally from both proximal $(<90 \mu \mathrm{m}$ radial distance from soma) and distal (>90 $\mu \mathrm{m})$ apical oblique segments, wherever staining permitted, (depicted in Figure 2). The spine density of each segment was calculated by dividing 
the total number of spines by the length of the corresponding segment. Spine types were determined on the basis of the ratio of the width $(\mathrm{W})$ of the spine head to the length ( $L$ ) of the spine neck and classified as (in $\mu \mathrm{m}$ ): Filopodia $(L>1.5)$, thin/long thin $(\mathrm{L}<1.5$ \& L:W $>1)$, stubby $(\mathrm{L}: \mathrm{W}<1)$, and mushroom $(\mathrm{W}>0.6)$ (Risher et al. 2014). In males, 1-8 segment samples $(10-20 \mu \mathrm{m})$ per animal from 2-5 neurons total were averaged to create a representative sample for each segment type per animal.

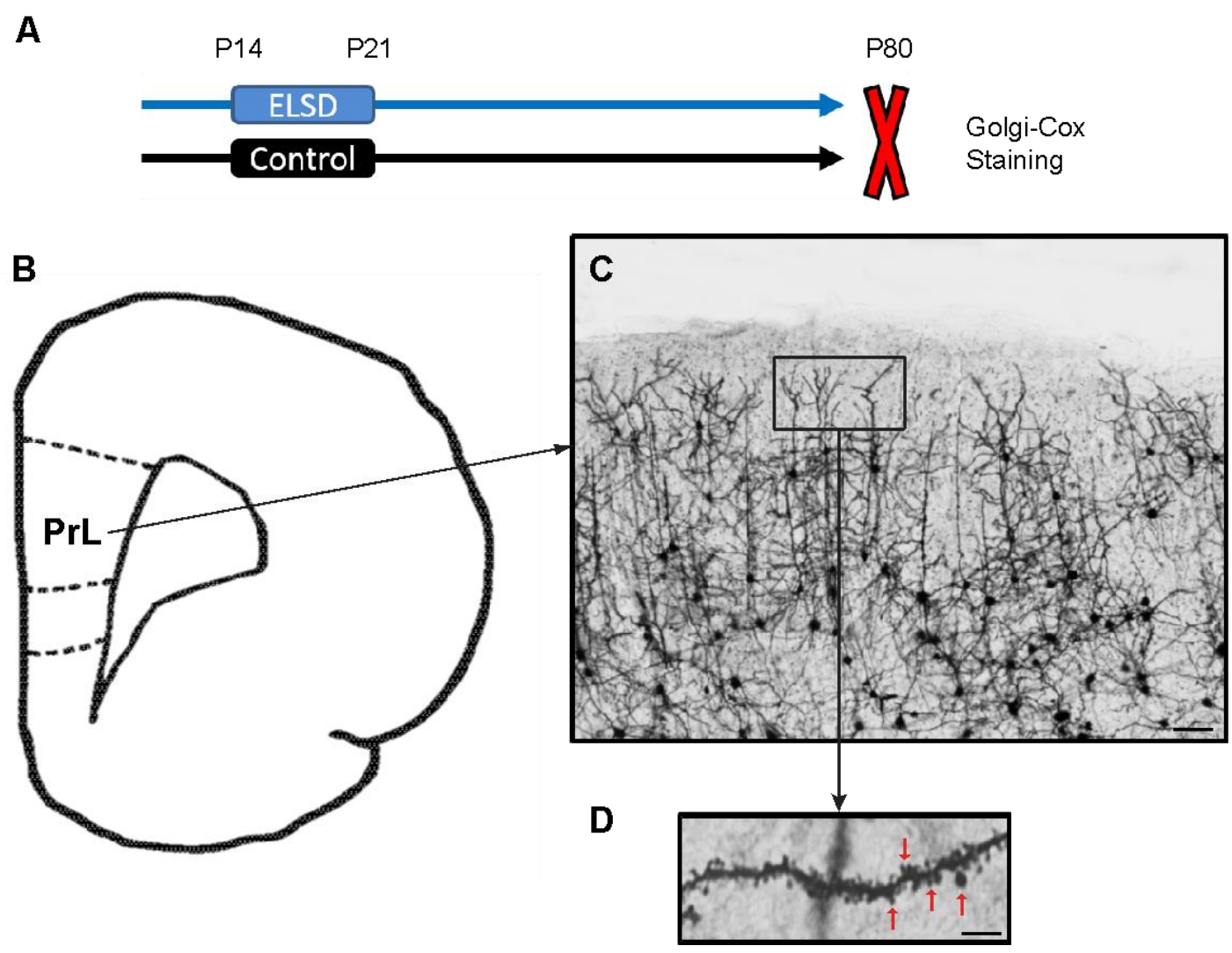

Figure 1: (A) Schematic timeline of the experimental protocol. (B) Schematic coronal section illustrating the prelimbic (PrL) cortex of the vole brain examined in Golgi preparations. (C) Golgi impregnated pyramidal neurons from layers II/II of the PrL, magnification 5x, scale bar, $70 \mu \mathrm{m}$. (D) Representative dendrite used to quantify spines (denoted by arrows), magnification 40x, scale bar, $10 \mu \mathrm{m}$ 




Figure 2: Schematic illustration of a cortical pyramidal neuron, denoting the various apical dendritic regions: oblique proximal $(<90 \mu \mathrm{m}$, blue), oblique distal $(>90 \mu \mathrm{m}$, red), and apical tufts (green). Basilar dendrites are not quantified (black).

\section{Statistical Analyses}

All statistical analyses were performed using SPSS v.24.0 (IBM Corp., Armonk, NY, USA). For all tests, $\mathrm{P} \leq 0.05$ was considered statistically significant. Differences in spine density, width, length and ratio of length to width were assessed using an independent samples t-test. All tests were two-tailed. Statistical analyses were performed using a multivariate ANOVA to assess possible differences in spine morphology classification. The values are shown as means \pm SEM, calculated based on one aggregate (i.e., average) per animal. Independent samples Kolmogorov-Smirnov test was used to determine probability distributions of spine length between groups. 


\section{RESULTS}

\section{Dendritic spine density, head width and length}

The average spine density in the apical distal $(>90 \mu \mathrm{m})$ oblique segments in layers II/III of the prelimbic (PrL) of ELSD males $(n=6)$ were $28 \%$ more dense than the control males $(n=4)$ (independent samples t-test, $t_{8}=2.381, P=0.045$ ). There were no significant differences between control $(n=4)$ and ELSD males $(n=4)$ in the apical tufts (independent samples $\mathrm{t}$-test, $\mathrm{t}_{6}=0.529, \mathrm{P}=0.618$,), nor were there differences between control $(n=4)$ and ELSD $(n=6)$ males in apical oblique dendrites located proximal (<90 $\mu \mathrm{m}$ ) to the soma (independent samples t-test, $\left.t_{8}=1.016, \mathrm{P}=0.339\right)$ (Figure $3 \mathbf{A}$ ). In the females (Figure 3B), the mean spine density on apical oblique dendrites located proximal to soma of pyramidal neurons in layers II/III of PrL of ELSD subjects $(n=5)$ was $10 \%$ more dense than that of the controls $(n=4)$. However, these differences did not achieve statistical significance (independent samples $t$-test, $t 7=1.894, P=0.10$ ). There were no significant differences between control $(n=3)$ and $\operatorname{ELSD}(n=5)$ females in the apical tufts (independent samples $t$-test, $t_{6}=0.569, P=0.590$ ), nor were there differences between control $(n=3)$ and ELSD $(n=4)$ females in apical oblique dendrites located distal (>90) to the soma (independent samples $t$-test, $t_{5}=0.772, P=0.475$ ) (Figure 3B).

Notably, the dendritic spines on apical oblique dendrites located distal to soma from ELSD males $(n=6)$ were $16 \%$ narrower than control males $(n=4)$ (independent samples $t$-test, $\left.t_{8}=3.701, P=0.006\right)$. There were no significant differences between control $(n=4)$ and ELSD males $(n=4)$ in the apical tufts (independent samples $t$-test, $t_{6}=1.821$, $P=0.119)$, nor were there differences between control $(n=4)$ and ELSD $(n=6)$ males in apical oblique dendrites located proximal to the soma (independent samples t- 
test, t8=1.016, $P=0.339$ ) (Figure. 3C). There were no significant differences between

control $(n=3)$ and ELSD $(n=5)$ females in the apical tufts (independent samples t-

test, $\left.t_{6}=0.706, P=0.507\right)$, and between control $(n=3)$ and $\operatorname{ELSD}(n=4-5)$ females in apical oblique dendrites located proximal $(<90 \mu \mathrm{m})$ and distal $(>90 \mu \mathrm{m})$ to the soma respectively (independent samples t-test, $t_{7}=0.123, P=0.172$; independent samples ttest, t5=0.626, $P=0.559$ ) (Figure 3D).

A

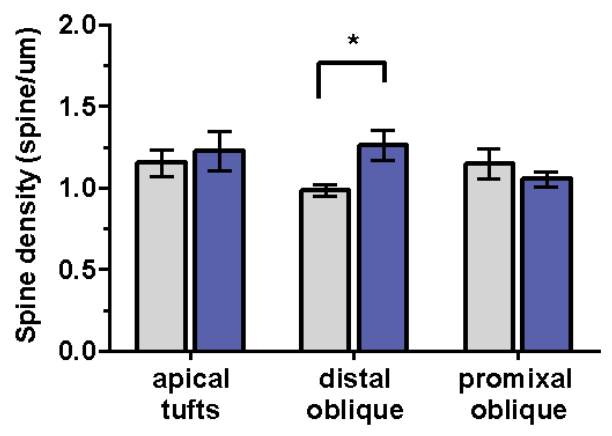

C

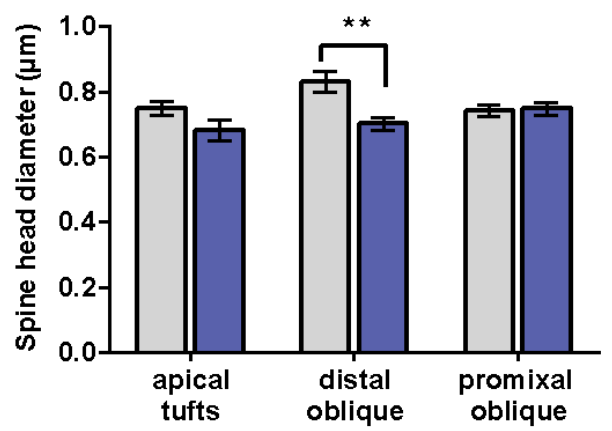

B

Females

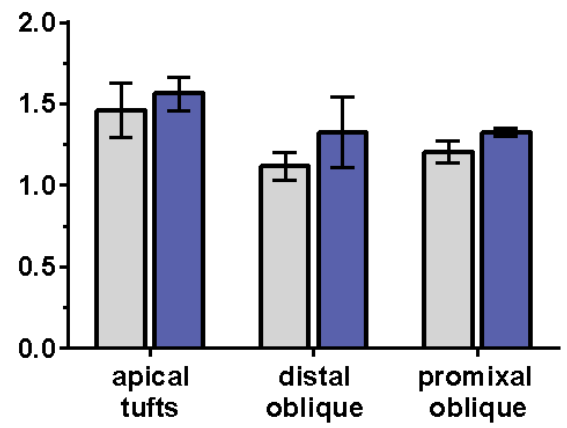

D

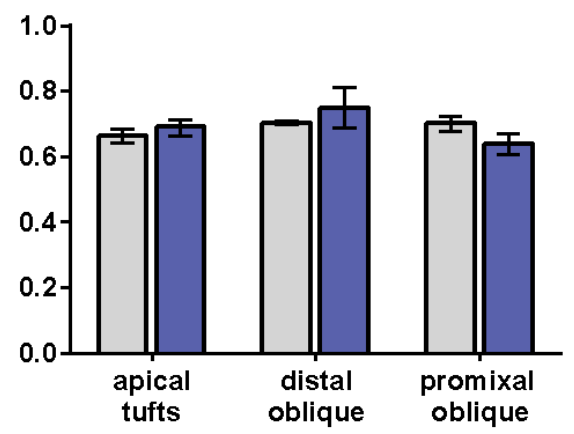

Figure 3: Comparison of spine head diameter and spine density in cortical pyramidal neurons in layers II/III of the PrL cortex.(A) Average spine density was increased significantly in the apical oblique dendrites located distal $(>90 \mu \mathrm{m})$ to the soma of ELSD males compared to control males. (B) No group differences found in females. (C) Spine head diameter was increased significantly in the apical oblique dendrites located distal $(>90 \mu \mathrm{m})$ to the soma of ELSD males compared to control males. (D) No group differences found in females. Data in bar graphs are presented as mean \pm s.e.m. 2-8 segment samples (10-20 $\mu \mathrm{m})$ per animal from 2-5 neurons total averaged to create a representative sample for each segment type per animal. ${ }^{*} \mathrm{P} \leq 0.05^{* *} \mathrm{P} \leq 0.01$. 
In addition to the investigations of the spine density and spine width of the pyramidal neurons of the PrL (layers II/III), analysis was also conducted on the mean length of spines, which revealed no significant difference control $(n=9)$ and $\operatorname{ELSD}(n=11)$ groups (independent samples $t$-test, $\mathrm{t}_{6}=0.732, \mathrm{P}=0.348$ ) Male and female subjects were combined for each group as there were no sex differences. Furthermore, the respective cumulative frequency distribution of the spine length for each dendritic segment between controls $(n=73)$ and ELSD subjects $(n=102)$ did not show a significant difference (independent samples Kolmogorov-Smirnov test, $\mathrm{P}=0.136$ ).

A

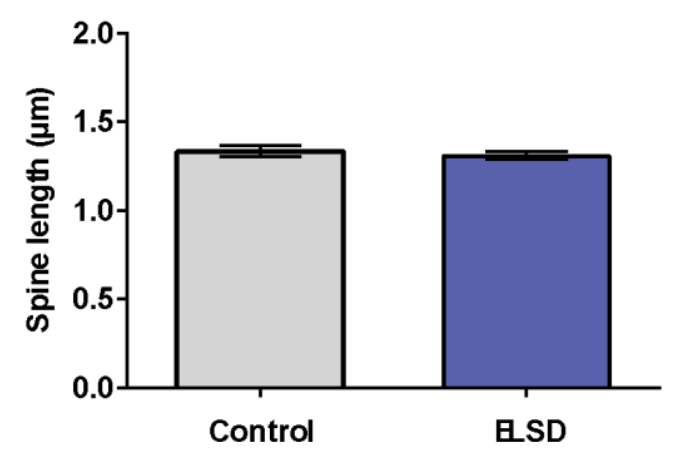

B



Figure 4: Comparison of spine length in cortical pyramidal neurons in layers II/III of the PrL cortex. (A) No significant differences in spine length between ELSD and control groups. 28 segment samples $(10-20 \mu \mathrm{m})$ per animal from 2-5 neurons total averaged to create a representative sample for each segment type per animal. (B) The cumulative frequency distribution of the spine length measures showed no significant difference. Data in the bar graphs are presented as mean \pm s.e.m. Data in the line graphs are the average of dendritic spine length for each segment. $\mathrm{P} \leq 0.05$

\section{Ratio of spine length to head diameter and morphological classification of dendritic spines}

On average, ESLD males $(n=6)$ had a $11.3 \%$ greater dendritic spine length to head diameter ratio (LDR) compared with the controls $(n=4)$ in the apical distal $(>90 \mu m)$ 
oblique segments in layers II/III of the PrL (independent samples t-test, t8=3.115, $P=0.014)$. There were no significant differences between control $(n=4)$ and ELSD males $(n=4)$ in the apical tufts (independent samples $t$-test, $t_{6}=0.529, P=0.618$ ), nor were there differences between control $(n=4)$ and $\operatorname{ELSD}(n=6)$ males in apical oblique dendrites located proximal $(<90 \mu \mathrm{m})$ to the soma (independent samples $t$-test, $t_{8}=1.016, P=0.339$ ) (Figure 5A). Interestingly, ELSD females $(n=5)$ had $8.4 \%$ greater spine LDR than controls $(n=4)$ (independent samples t-test, t8=2.364, $P=0.05)$. There were no significant differences between control $(n=3)$ and $\operatorname{ELSD}(n=5)$ females in the apical tufts (independent samples $\mathrm{t}$-test, $\mathrm{t}_{6}=0.1 .53, \mathrm{P}=0.176$,), nor were there differences between control $(n=3)$ and ELSD $(n=4)$ females in apical oblique dendrites located distal $(>90)$ to the soma (independent samples t-test, $t_{5}=0.626, P=0.559$ ) (Figure 5B). Figure 5C shows that the overall increase of spine LDR seen in the apical distal $(>90 \mu \mathrm{m})$ oblique segments of ELSD males ( $n=6$ ) (Figure $5 \mathbf{A}$ ) is primarily due to a selective significant increase in the total proportion of thin spines (multivariate ANOVA, $F=5.854, P=0.040$ ), with no statistically significant difference in mushroom or other spines $(P>0.1)$. There were no significant differences in long thin or other spines between ELSD $(n=5)$ and control $(n=4)$ females in apical oblique dendrites located proximal $(<90)$ to the soma (multivariate ANOVA, $\mathrm{P}>0.2$ ) (Figure. 5D). 


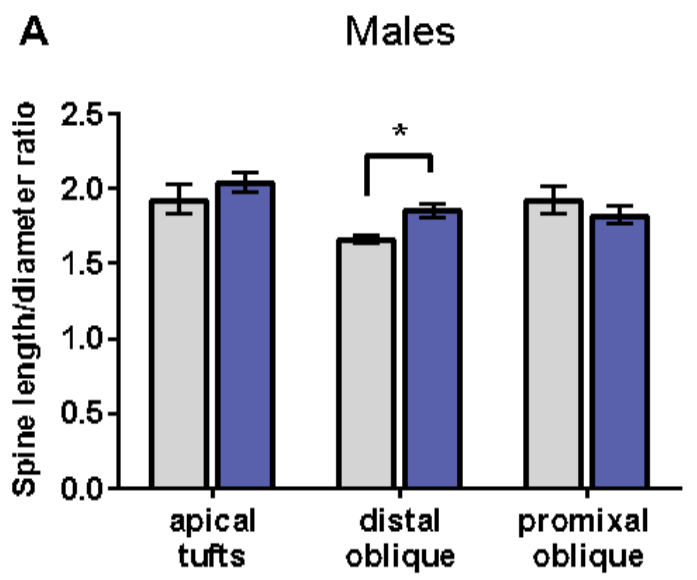

B

Females

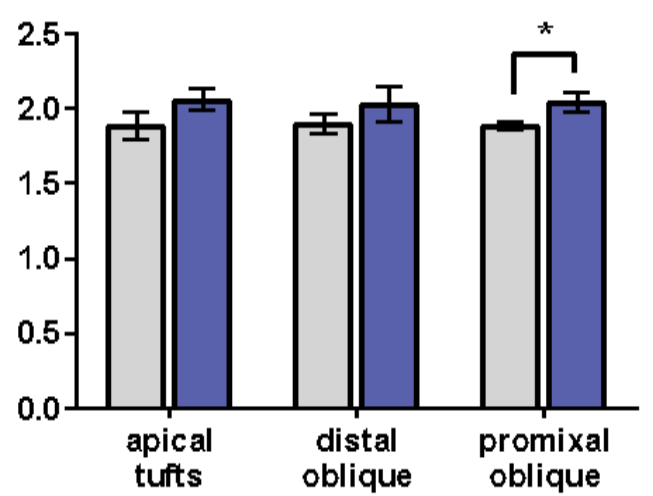

C Males - Distal Oblique

D

Females - Proximal Oblique
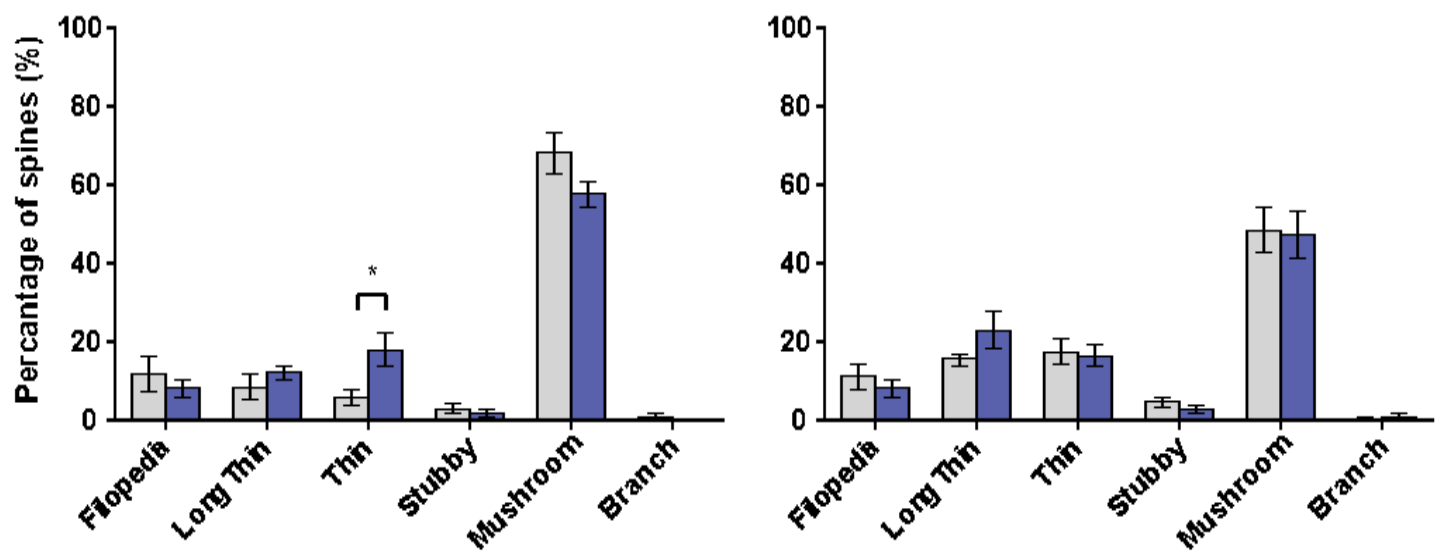

Figure 5: Morphological analysis of dendritic spines in layers II/III of PrL pyramidal neurons. (A) Dendritic spine length to head diameter ratio (LDR) was increased significantly in the distal oblique segments of ELSD males. (B) Spine LDR was increased significantly in the proximal oblique segments of ELSD females. (C) Thin spines significantly increased in ELSD males. (D) No significant group differences in females. Data in bar graphs are presented as mean \pm s.e.m. 2-8 segment samples $(10-20 \mu \mathrm{m})$ per animal from 2-5 neurons total averaged to create a representative sample for each segment type per animal. ${ }^{*} \mathrm{P} \leq 0.05$

\section{DISCUSSION}

The aim of this study was to compare the dendritic spine density and spine morphology in the prelimbic region of the prefrontal cortex (PFC), regions implicated in autism neuropathology, in early life sleep disrupted (ELSD) prairie voles versus 
undisturbed control animals. Recent studies have postulated that sleep plays a major role in synaptic plasticity and pruning of dendritic spines (Bushey et al., 2011; Frank et al., 2001; Maret et al., 2011; Tononi and Cirelli, 2014). Here we demonstrate that in ELSD male prairie voles, spine density and spine width selectivity increased in the apical oblique dendrites segments located distal to the soma. In contrast, ELSD females showed no differences in any spine density, width and length parameters across the various apical dendritic regions (depicted in Figure 2). Overall, these findings suggest that early life REM sleep may play a critical role in synaptic plasticity, and enhance our understanding of the neuropathology of ASD symptomatology.

\section{Spine density and morphometric features in PFC layers II/III}

Developmental alterations of excitatory synapses are implicated in autism spectrum disorders (ASDs). Hutsler and Zhang (2010) observed increased spine density in the frontal lobe of ASD post-mortem brains. Recent studies have increasingly shown that alterations in spine dynamics and synaptic efficacy are modulated by sleep and sleep loss (Raven et al., 2017; Tononi and Cirelli, 2014). In particular, REM sleep is important for pruning and maintaining new synapses formed during adolescence and into adulthood (Li et. al, 2016).

We assessed spine density in adult prairie voles and found a selective increase in the apical oblique dendrite segments located distal to the soma in layers II/III of the PFC pyramidal neurons in ELSD males. No differences were found in ELSD females and controls. The density of dendritic spines has direct implications for the functionality and connectivity of the cell. Synapses tend to be located at the heads of dendritic 
spines, therefore higher spine densities would suggest a higher density of synapses.

Furthermore, in ELSD males, the more distal aspect of apical oblique dendritic segments exhibited the most pronounced alterations in two of the three dendritic spine morphometric parameters (width and ratio of spine length to width). The overall increase in spine length to width ratio seen in the apical distal oblique segments of ELSD males is primarily due to a selective significant increase in the total proportion of thin spines. The excessive amount of thin immature spines, is one of the spine morphological characteristics of ASD (Martínez-Cerdeño, 2017). The increased ratio of spine length to width in the proximal oblique segments of ELSD females is interesting, although there were no significant differences in spine types between groups. Together, this results support the notion that sleep and sleep loss are linked to spine dynamics and synaptic plasticity and may underlie aberrant spine growth in developmental disorders in humans.

\section{Pyramidal-neuron structure and domains of synaptic input}

Layer II/III pyramidal neurons are the major excitatory neurons of the prefrontal cortex that receive local and distant cortical projections (Spruston, 2008; Little and Carter, 2013). Pyramidal neurons are characterized by separate apical and basal dendritic trees. (Figure 2). Several basal dendrites extend from the soma and branch several times before terminating. Usually, a single large apical dendrite emerges from the apex of the pyramidal soma and bifurcates at variable distances to form two main apical dendrites and continue to extend and branch towards the pial surface, giving rise to the apical tufts. Oblique apical dendrites stem from the main apical dendrite prior to the bifurcation of the main branch. During our analyses of spine density and 
morphology, apical oblique dendritic regions $<90 \mu \mathrm{m}$ from the soma were regarded as proximal dendrites, whereas the further apical oblique dendritic regions $>90 \mu \mathrm{m}$ were regarded as distal dendrites (Figure 2). The rationale for this classification is based on nonuniform synaptic integration (Branco and Häusser, 2011) and potential spine density and spine head dynamic changes as a function of distance from the soma (BenavidesPiccione et al., 2002). Both basal dendrites and proximal apical dendrites receive excitatory inputs from local cortical projection (Spruston, 2008), thus we forgo the analysis of basal dendrites for the current set of experiments. Apical tufts receive excitatory synaptic inputs from further cortical and thalamic locations (Spruston, 2008). In ELSD males, the increase in distal apical oblique spine density, driven by an increase in thin immature spines, suggests hypo-connectivity of long-range cortico-cortical and cortico-thalamic circuits (Just et al., 2004) and may underlie an imbalance in excitation/inhibition (Sporns et al., 2001; Gogolla et al., 2009). Surprisingly, no differences were observed in the proximal apical oblique dendrites of ELSD males, as enhanced local connectivity (Belmonte et al., 2004) is generally a feature of ASD, and develops together with low long-distance connectivity (Just et al., 2004).

\section{Neurobiology of social bonding in prairie voles}

Impaired social interaction is a hallmark of neurodevelopmental disorders, including ASD. The prairie vole is unique among rodent models for their characteristic highly social behavior, including the ability to form pair bonds with potential mates (Young and Wang, 2004). The prefrontal cortex (PFC) is a late-maturing region that 
modulates pair bonding (Young and Wang, 2004), possibly through the interplay between excitatory and inhibitory neurotransmission, thought to be relevant in disorders such as ASD (Yizhar, et al., 2011). Further, several candidate neurotransmitters systems (i.e., oxytocin and vasopressin) that were first discovered to regulate vole social bonding have also been found relevant to human autism (Young and Wang, 2004; Insel et al., 1999). Given their unique patterns of social behavior that are relatively uncommon in non-human mammals, prairie voles have become excellent animal models for studying complex social behaviors. In addition, they are wild caught and outbred, resulting in a genetically diverse and behaviorally heterogeneous, which better captures a larger spectrum of individual differences in behavior.

Emerging research in our lab suggests that selectively suppressing REM sleep early in life in the socially monogamous prairie vole impairs pair bond formation and increases inhibitory interneurons in the PFC (Lim et al., unpublished data), consistent with ASD pathology. We found that impaired sleep-dependent social bonding in ELSD voles may potentially be associated with impaired pruning of dendritic spines in the PFC. Moreover, this synaptic pruning deficit is most profound in the males, reminiscent of the male bias seen in our ELSD model and also typically found in ASD. Previous human autism studies have shown that there are inherent sex differences in dendritic morphology and connectivity patterns (Hutsler and Zhang, 2010), therefore it is reasonable to suspect that a pattern of hyper-connectivity or hypo-connectivity observed in ELSD males may differ from ELSD females. It is worth noting that the female group sizes were quite small in this study $(n=3-5)$, thus adding more subjects could potentially yield significant group differences between control and ELSD females. 


\section{Role of REM sleep on spine pruning}

Early in development, neuronal connectivity is shaped by an initial abundance of immature spines and synapses, followed by a period maintaining stronger connections and pruning weaker connections. It has been suggested that synaptogenesis and pruning during development could be modulated by sleep and wakefulness (Tononi and Cirelli 2006). In particular, REM sleep has shown to be important for pruning and establishing stable synapses formed during adolescence and into adulthood (Li et. al, 2016). Our results are support these findings, as we found reduced synaptic pruning of spines, resulting in excessive and less stable spines in ELSD males which underwent selective REM deprivation using our method of sleep disturbance. Recent work by Fang et al. demonstrated overproduction of neurons was accompanied by dysregulated excitatory connections, resembling an autism behavior phenotype in mice. These findings, together with our results, potentially elucidate that sleep disturbances may directly contribute to spine density and alter synaptic connectivity underlying autistic social behavior and etiology of ASD.

\section{FUTURE DIRECTIONS}

While our preliminary study solely examined one specific region and layer within the PFC, we are interested in extending our study to other layers and regions within the PFC and other brain regions such as the S1 Barrel cortex and CA1 Hippocampus, which are relevant to autism neuropathology. Furthermore, little is known about the developmental trajectory of ELSD, thus it would be interesting to quantify dendritic spines in juvenile ELSD voles and compare them to adult ELSD voles to investigate 
possible developmental changes in neuronal connectivity. Additionally, more research is needed to analyze dendritic complexity and branching, as these measures may have implications for synaptic plasticity and neuropathology in ASD. 


\section{ACKNOWLEDGEMENTS}

I am most grateful to Miranda M. Lim, MD, PhD and Carolyn Jones, PhD for their supportive mentoring, guidance and helpful comments on this project and thesis. I also would like to thank Marina Guizzetti's lab for kindly providing their microscope and technical assistance. This work was supported by the VA Biomedical Laboratory Research \& Development (BLR\&D) Career Development Award (CDA) \# IK2 BX002712, Portland VA Research Foundation, Brain \& Behavior Foundation NARSAD Award, Collins Medical Trust, NIH EXITO Institutional Core \#UL1GM118964, and NIH T32 5T32AA7468-29. 


\section{REFERENCES}

Alaerts et al. (2016). Sex differences in autism: a resting-state fMRI investigation of functional brain connectivity in males and females. Social cognitive and affective neuroscience, 11(6), 1002-1016.

Bailey, C. and Kandel, R. (1993). Structural changes accompanying memory storage. Annual review of physiology, 55(1), 397-426.

Belmonte, M. et al. (2004). Autism and abnormal development of brain connectivity. Journal of Neuroscience, 24(42), 9228-9231.

Benavides-Piccione et al. (2002). Cortical area and species differences in dendritic spine morphology. Journal of neurocytology, 31(3-5), 337-346.

Branco, T., and Häusser, M. (2011). Synaptic integration gradients in single cortical pyramidal cell dendrites. Neuron, 69(5), 885-892.

Buonomano, V. and Merzenich, M. (1998). Cortical plasticity: from synapses to maps. Annual review of neuroscience, 21(1), 149-186.

Bushey, D. et al. (2011). Sleep and synaptic homeostasis: structural evidence in Drosophila. Science, 332(6037), 1576-1581.

Chang, FL and Greenough, WT (1984) Transient and Enduring Morphological Correlates of Synaptic Activity and Efficacy Change in the Rat Hippocampal Slice. Brain Research 309(1): 35-46.

Fang, W. et al. (2014). Overproduction of upper-layer neurons in the neocortex leads to autism-like features in mice. Cell reports, 9(5), 1635-1643.

Fiala JC (2005). Reconstruct: a free editor for serial section microscopy. Journal of Microscopy 218:52-61. 
Frank, M. et al. (2001). Sleep enhances plasticity in the developing visual cortex. Neuron, 30(1), 275-287.

Gray, EG (1959). Axo-Somatic and Axo-Dendritic Synapses of the Cerebral Cortex: An Electron Microscope Study. Journal of Anatomy 93: 420-433.

Harris, KM and Kater SB (1994). Dendritic Spines: Cellular Specializations Imparting Both Stability and Flexibility to Synaptic Function. Annual Review of Neuroscience 17: 341-371.

Harris, KM and Stevens, JK (1989) Dendritic Spines of CA1 Pyramidal Cells in the Rat Hippocampus: Serial Electron Microscopy with Reference to Their Biophysical Characteristics. The Journal of Neuroscience: The Official Journal of the Society for Neuroscience 9(8): 2982-2997.

Haws, ME et al. (2014). PTEN Knockdown Alters Dendritic Spine/Protrusion Morphology, Not Density. Journal of Comparative Neurology 522(5): 1171-1190.

Hutsler, J. and Zhang, H. (2010). Increased dendritic spine densities on cortical projection neurons in autism spectrum disorders. Brain research, 1309, 83-94.

Insel, T et al. (1999). Oxytocin, vasopressin, and autism: is there a connection?. Biological psychiatry, 45(2), 145-157.

Just, M. et al. (2004). Cortical activation and synchronization during sentence comprehension in high-functioning autism: evidence of underconnectivity. Brain,127(8), 1811-1821.

Kasai, H. et al (2003). Structure-Stability-Function Relationships of Dendritic Spines. Trends in Neurosciences 26(7): 360-368.

Li, Y et al. (2014) Effects of Chronic Sleep Fragmentation on Wake-Active Neurons and 
the Hypercapnic Arousal Response. Sleep 37(1): 51-64.

Little, J. and Carter, A. (2013). Synaptic mechanisms underlying strong reciprocal connectivity between the medial prefrontal cortex and basolateral amygdala. Journal of Neuroscience, 33(39), 15333-15342.

Maret, S et al. (2011). Sleep and Waking Modulate Spine Turnover in the Adolescent Mouse Cortex. Nature Neuroscience 14(11): 1418.

Martínez-Cerdeño, V. (2017). Dendrite and spine modifications in autism and related neurodevelopmental disorders in patients and animal models. Developmental neurobiology, 77(4), 393-404.

McGraw, LA and Young, LJ (2010). The prairie vole: an emerging model organism for understanding the social brain. Trends in Neurosciences, 33(2), 103 Nimchinsky et al. (2002). Structure and function of dendritic spines. Annual review of physiology, 64(1), 313-353.

Paus, T. Keshavan, M and Giedd. JN (2008) Why Do Many Psychiatric Disorders Emerge during Adolescence? Nature Reviews Neuroscience 9(12): 947.

Paxinos, G. and Franklin, K. (1997) The Mouse Brain in Stereotaxic Coordinates. Academic Press, San Diego

Rakic, P., J. P. Bourgeois, and P. S. Goldman-Rakic (1994). Synaptic Development of the Cerebral Cortex: Implications for Learning, Memory, and Mental Illness. Progress in Brain Research 102: 227-243.

Raven et al. (2017). The role of sleep in regulating structural plasticity and synaptic strength: Implications for memory and cognitive function. Sleep medicine reviews. 
Risher, CW et al (2014). Rapid Golgi Analysis Method for Efficient and Unbiased Classification of Dendritic Spines. PLoS ONE

Rochefort, NL. and Konnerth A. (2012). Dendritic Spines: From Structure to in Vivo Function. EMBO Reports 13(8): 699-708.

Roffwarg, HP et al. (1966). Ontogenetic Development of the Human SleepDream Cycle. Science 152(3722): 604-619.

Segal et al (2014). Dendritic Spines: The Locus of Structural and Functional Plasticity. Physiological Reviews 94(1): 141-188.

Siegel, J. M. (2005). Clues to the functions of mammalian sleep. Nature, 437(7063), 1264.

Spruston, N. (2008). Pyramidal neurons: dendritic structure and synaptic integration. Nature Reviews Neuroscience, 9(3), 206.

Tononi, G. and Cirelli C. (2006). Sleep Function and Synaptic Homeostasis. Sleep Medicine Reviews 10(1): 49-62.

Tononi, G. and Cirelli C. (2014) Sleep and the price of plasticity: from synaptic and cellular homeostasis to memory consolidation and integration." Neuron 81.1: 1234.

Trachtenberg JT et al (2002) Long-Term in Vivo Imaging of Experience-Dependent Synaptic Plasticity in Adult Cortex. Nature 420(6917): 788-794.

Yizhar, et al. (2011). Neocortical excitation/inhibition balance in information processing and social dysfunction. Nature, 477(7363), 171.

Young, L. and Wang, Z. (2004). The neurobiology of pair bonding. Nature neuroscience, 7(10), 1048. 